\title{
CAVITATION EROSION PREDICTION ON FRANCIS TURBINES-PART 1 MEASUREMENTS ON THE PROTOTYPE
}

\author{
P. BOURDON, M.FARHAT, R. SIMONEAU \\ Hydro-Québec \\ 1800 boul. Lionel Boulet, Varennes, Québec, Canada, J3X ISI \\ F. PEREIRA, P. DUPONT., F. AVELLAN \\ $I M H E F / E P F L$ \\ 33 av. de Cour, CH 1007 Lausanne, Switzerland \\ J.-M. DOREY \\ Electricité de France \\ 6, Quai Watier, 78401 CHATOU Cédex, France
}

\section{Abstract}

In the process of developing tools for cavitation erosion prediction of prototypes from model tests, 4 on board aggressiveness evaluation methods were tested on a severely eroded blade of a $266 \mathrm{MW}$ Francis turbine. These are pressure, pit counting, DECER electrochemical and vibration measurements. All methods provided coherent results on the blade mounted measurements. The test program provided understanding of the heterogeneous erosion distribution of the prototype blades and quantitative data for comparison in subsequent tests on the model of the machine.

\section{Introduction}

The prediction of cavitation erosion of a prototype turbine from model tests requires that measurement tools be available to characterize the aggressiveness of the cavitating flow in both scales. To develop such tools, IMHEF, Electricité de France and Hydro-Québec pooled their resources in an ambitious research program involving various measurement techniques both on the prototype and on the model of a $266 \mathrm{MW}$ Francis turbine with a well documented cavitation erosion history. Preliminary measurements on this prototype (1) had proven to be incomplete but also very encouraging. A more ambitious test program was conceived with improvements in the array of sensors utilized, sample mounting methods, data acquisition systems and hydraulic test conditions. The test program was divided in two parts, the first with pressure sensors mounted on the suction side of blade \#4, a well eroded blade, to identify the type of cavitation present and its aggressiveness in terms of pressure pulses. In the second, polished metallic samples were mounted in place of the sensors for pitting studies. This experimentation program took place in June 1995. 


\section{Test set-up}

\subsection{MECHANICAL INSTALLATIONS}

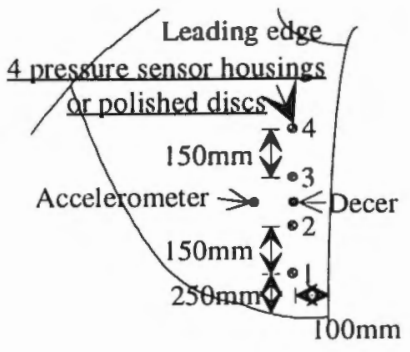

Figure I. Blade 4 sensor and disc positions

This machine has the particularity that the erosion is much worse on about half of the blades and is localized towards the trailing edge near the blade to band fillet. Blade \#4 was retained for testing as it had been used in the preceding tests because of its severe erosion history. Consequently, six $45 \mathrm{~mm}$ diameter holes were counterbored in this blade as shown in Figure 1 on the low pressure side. An intricate drilling and shimming scheme was developed by IMHEF to insure perpendicularity of the hole axis with the complex blade surface and optimal flush mounting of the various sensor housings. All housings or sample mounting supports were securely bolted down to the blade from the pressure side. Figure 2 shows the magnetic drill mounting base positioned in place as well as some of the already bored holes.

Holes were also bored above the draft tube access door to mount a dynamic pressure sensor, pass cables for 5 underwater accelerometers and cables to immersed spark plugs for the dynamic calibration of the set-up in watered conditions. A cylinder

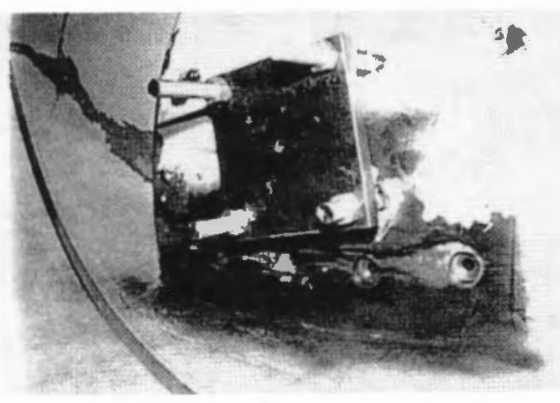

Figure 2. Drill mounting base housing the custom designed onboard data acquisition electronics was welded in place in the runner nose cone. Cables were run from the sensors to this unit through a stainless steel tube welded to the pressure side of bade 4 and covered with an hydraulically smooth buildup of resin to minimize turbulence. Signal and control cables were then brought out to the stationary world through the hollow turbine-alternator shaft using the air injection path and a double slip ring arrangement fixed to the end of the alternator exciter shaft. A threaded hole was also machined at the lower turbine guide bearing to receive a piezoelectric force exciter for sinusoidal sweep calibration of the transmissibility function between the guide bearing and 5 underwater accelerometers mounted on the runner blades.

\subsection{SENSORS}

For the pressure tests, 4 of the blade holes (\# 1-4) received stainless steel sensor housings bearing each 4 piezoresistive pressure sensors allowing to measure both static and dynamic pressures. Each housing contained 2400 bar and 21000 bar Keller sensors. These housings were replaced with optically polished $316 \mathrm{~L}$ stainless steel 
discs for the pitting tests. The two remaining holes were occupied respectively by a DECER electrochemical cavitation erosion sensor and a $4 \mathrm{kHz}$ bandwidth damped piezoresistive accelerometer. These were used in both the pressure and pitting tests.

In addition to the onboard sensors mentioned above, four high frequency accelerometers monitored the cavitation impacts at the lower guide bearing while another was installed above the draft tube access door along with a Kistler 701 wide band dynamic pressure sensor. A tachometric probe allowed to synchronize data acquisition on multichannel wide band data acquisition systems. The test program included calibration of the setup through the measurement of transmissibility functions both in air and in water between the blades and measurement points at the lower guide bearing. This step is required to determine absolute cavitation aggressiveness levels from remotely measured vibration or pressure data. For this purpose, 5 accelerometers were mounted on blades $3,4,710$ and 13 on the suction side at a position corresponding to the location of the DECER probe on blade 4 shown in Figure 1. Direct and reciprocity impact and direct spark generated bubble calibration techniques were utilized. Two underwater spark plugs were used to generate bubbles at two locations close to the suction side of blade 4 . A sine sweep technique was used in a reciprocity mode by exciting the structure with controlled force at the lower guide bearing.

\subsection{DATA ACQUISITION SYSTEMS}

For the purpose of this program, IMHEF designed an onboard data acquisition system with two variable gain $100 \mathrm{kHz}$ bandwidth data channels and two front end remotely controlled channel multiplexers. This system allowed to scan the 16 blade mounted pressure sensors as well as the $4 \mathrm{kHz}$ bandwidth accelerometer. Signals were converted from voltage to frequency for transmission through the dual slip ring arrangement to avoid noise pickup in the alternator

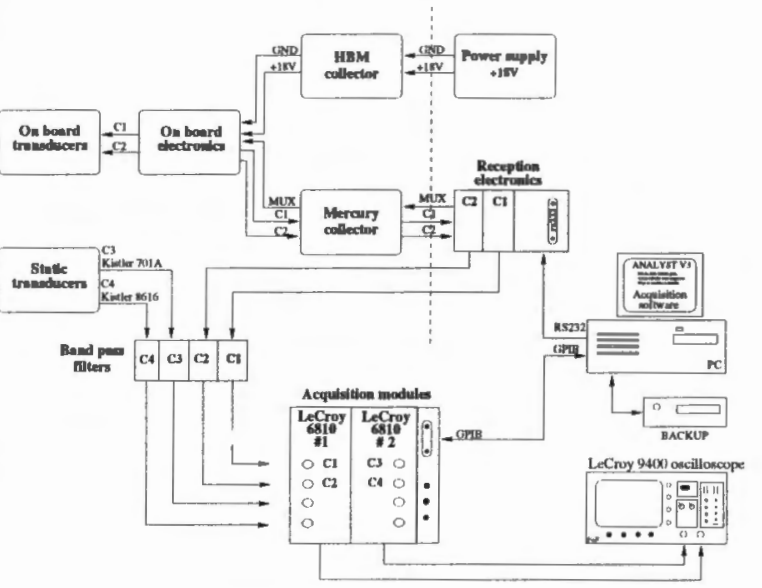

Figure 3. IMHEF data acquisition system. environment. The signals were then converted back to voltage with a frequency to voltage converter. Extra tracks on the slip rings were used to carry an RS 232 control link, the DECER electrochemical work and auxiliary potentials and the power supply current for the onboard electronics.

Data acquisition was assured by two separate systems provided by IMHEF and Hydro-Québec. The first system is based on 2 LeCroy 6810 acquisition modules configured to acquire each 2 input signals with 12 bit resolution at sampling frequencies up to $5 \mathrm{MHz}$. A 1 Mbyte memory in each module is shared between the 
two input channels. The acquisition is controlled via a GPIB port by a PC running Analyst software developed at IMHEF in the ASYST environment. On line monitoring of signals from this system is performed with a LeCroy DO9400 oscilloscope. Signals are first passed through antialiasing filters before acquisition. This system is illustrated in Figure 3.

The second includes a 9 channel HP3565S 13 bit $100 \mathrm{kHz}$ bandwidth data acquisition system controlled by LMS Fourier Monitor software, a Nicolet 5004 channel 12 bit $10 \mathrm{Mhz}$ digitizer with a 1 megasample memory on each channel and a Sony PC216A 16 bit resolution digital tape recorder used in an 8 channel $10 \mathrm{kHz}$ bandwidth configuration.

Both systems were utilized for the dynamic calibration of the test setup and for acquisition during the tests. The instrumentation was completed with a bank of programmable bandwidth and gain analog filters and envelope detectors. A potentiostat circuit conditioned the DECER signal which was recorded on an analog chart recorder as well as on the SONY tape recorder. An audio amplifier and loudspeaker combination allowed to assess audibly on site the impulsive nature and the intensity of the cavitation signals perceived by the blade mounted accelerometer.

\section{Test conditions}

Conditions for the pressure and pit counting tests appear in the following table.

TABLE 1. Test conditions

\begin{tabular}{|c|c|c|c|c|}
\hline $\begin{array}{c}\text { Test } \\
\text { Identification }\end{array}$ & $\begin{array}{c}\text { Guide vane opening } \\
(\%)\end{array}$ & $\begin{array}{c}\text { Power } \\
(\mathrm{MW})\end{array}$ & $\begin{array}{c}\text { Downstrearn level } \\
(\mathrm{m})\end{array}$ & $\begin{array}{c}\text { Duration } \\
\text { (h.min) }\end{array}$ \\
\hline \multicolumn{5}{|c|}{ Pressure tests } \\
\hline I & 78 & 228 & 205.7 & 6.0 \\
\hline 2 & 90 & 259 & 205.8 & 3.0 \\
\hline 3 & 78 & 228 & 207.4 & 4.0 \\
\hline 4 & 90 & 255 & 207.6 & 2.15 \\
\hline & 78 & 227 & 206.2 & .41 \\
\hline M1 & 78 & 223 & 207.4 & .41 \\
\hline M2 & 90 & 253 & 207.5 & .40 \\
\hline M3 & 85 & 247 & 205.7 & .17 \\
\hline M4-1 & 80 & 234 & 205.6 & .16 \\
\hline M4-2 & 75 & 219 & 205.5 & .15 \\
\hline M4-3 & 70 & 201 & 205.5 & .15 \\
\hline M4-4 & 72 & 206 & 205.9 & .40 \\
\hline M5 & 75 & & \\
\hline
\end{tabular}

In order to evaluate the effect of the variation of the cavitation index on the cavitation development, tailwater levels were raised in the downstream reservoir over a two week period to allow testing of the machine with high downstream levels with all available machines operating. These conditions had not been possible in the preliminary program (1). Low tailwater testing was done by operating only the test unit in the powerhouse. 


\section{Test results}

\section{I CALIBRATION}

Before running the operating tests on the prototype, an extensive calibration program was performed to characterize the transmissibility function (ratio of output acceleration power spectrum to input force power spectrum) between the attacked areas on the blades and the monitoring points either on the blades or at the lower guide bearing. Measurements were performed with the runner in air and then in water. The linearity

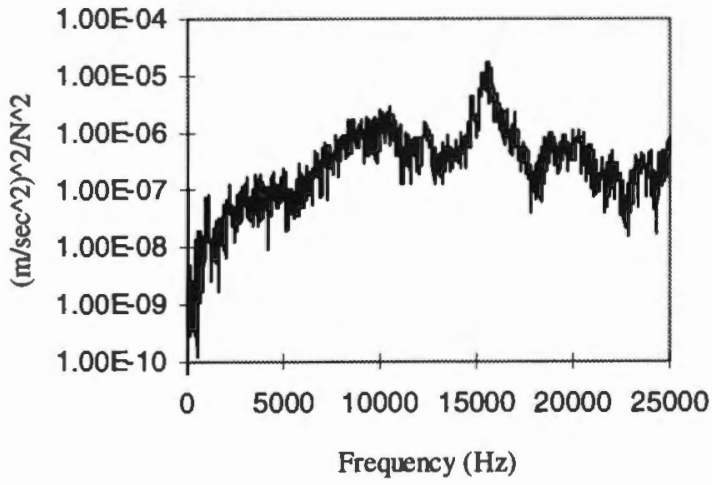

Figure 4. Lower guide bearing to blade transmissibility. of the structure was verified by reciprocity measurements using instrumented hammer excitation techniques with the machine in air. The average transmissibility function with the runner under water was then evaluated by impacting at the lower guide bearing monitoring point at 90 degrees from upstream on the spiral case upstream side and measuring simultaneously the response on 5 blades. The result of this measurement is shown in Figure 4. This function was then used to infer acting forces on the blades from acceleration measurements during subsequent testing.

\subsection{PRESSURE TESTS}

The first pressure test performed with a low tailwater level produced cavitation conditions on blade 4 such that violent implosions were localized in the area of the two downstream pressure sensor housings. This was later confirmed in pitting test M1 under similar conditions and by the severe marking of the stainless steel sensor housings after the pressure tests. As a result, the 8 sensors in the most downstream positions and 2 more on the next upstream housing were progressively put out of service as the time exposure increased. The remaining sensors exhibited a main
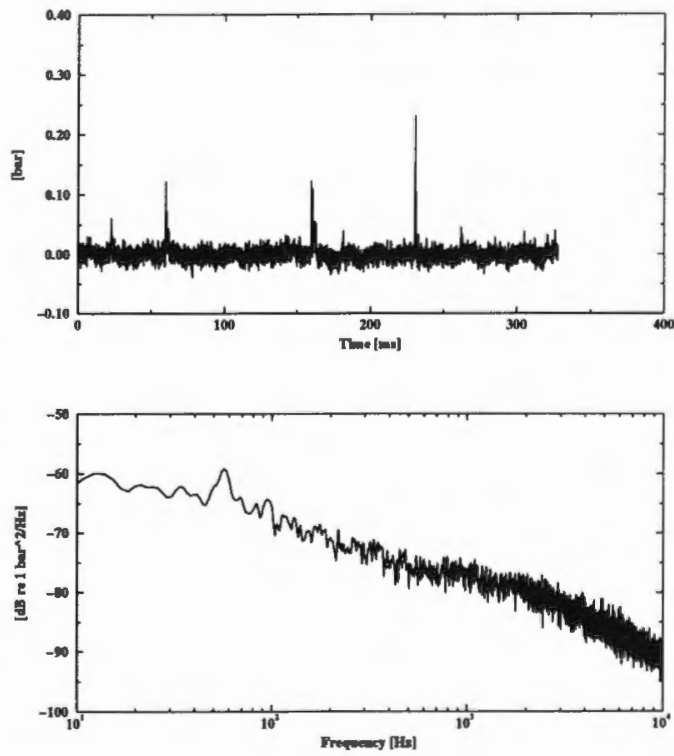

Figure 5. Typical pressure sensor time trace and average Power Density Spectrum 
component in their power spectral density spectra around $60 \mathrm{~Hz}$ which corresponds to the guide vane passing frequency for a rotating observer. An example is given in Figure 5. This result suggests the existence of a cavitation cloud whose pulsation is modulated by the pressure variations generated by passing in front of the guide vanes. This in turn commands forced vortex shedding in the closure area of this cloud and impacts on blade 4 at this rate. This is confirmed by the envelope analysis of the high frequency acceleration of the blade which shows a strong component at or near this frequency. In pressure test \#3, this hypothesis was supported by the remaining upstream sensors which were still operational. As the downstream level was raised, the cavitation cloud moved in the upstream direction in such a way that sensors on. housing 4 measured vapor pressure while those on housing 3 showed intense activity with enormous fluctuations which occasionally saturated the frequency to voltage converter. The pitting tests confirmed these indications as disc 3 was the most severely marked under the same hydraulic conditions.

\subsection{PITTING TESTS}

\subsubsection{Pit counting}

The 1993 test program had seen the most exposed glued discs torn away in the flow and the impossibility of testing with high downstream levels to evaluate the effect of this parameter on the cavitation development. As mentioned earlier, these two elements were corrected in this program. After the pressure tests, the pressure sensor housings were replaced with polished disc holders bolted down to the blade and the discs were replaced after each of the roughly 40 minute exposure periods. An exception to this was made in test M4 which consisted of 4 successive 15 minute exposures at $85,80,75$ and $70 \%$ guide vane opening in one sequence with a low downstream level. Three of the pressure test conditions were repeated, the $90 \%$ low downstream level test was skipped as it had been performed in 1993, the sweep test was added and was followed by a final point at $72 \%$ guide vane opening.

The results of these tests are summarized in Figure 6 which shows all of the disc samples along with a $10 \mathrm{X}$ micrograph of typical pitted areas on those discs that were marked by the cavitation impacts. The size of the pits can be appreciated by the $1 \mathrm{~mm}$ references which are shown below samples $2 \mathrm{M} 1$ and $3 \mathrm{M} 2$. The first digit in the sample number corresponds to its position on the blade (see Fig. 1). Downstream is left and upstream right. At the left is indicated the guide vane opening and downstream level for each test. From this Figure it can be clearly seen that the largest pits are observed near the trailing edge of the blade in tests M1 and M4 with the low downstream level. Smaller pits occur on discs 3 or 2 when the downstream level is raised as in test M2 or the guide vane opening is reduced as in test M5. Opening the guide vanes to $90 \%$ has for effect to move the pitting area to disc 4 towards the trailing edge or even beyond as no pits are observed on upstream discs 2 to 4 . The sweep test shows the worst pitting on disc 2 , the result of the combined marking during the 80 and $75 \%$ guide vane opening exposure. 


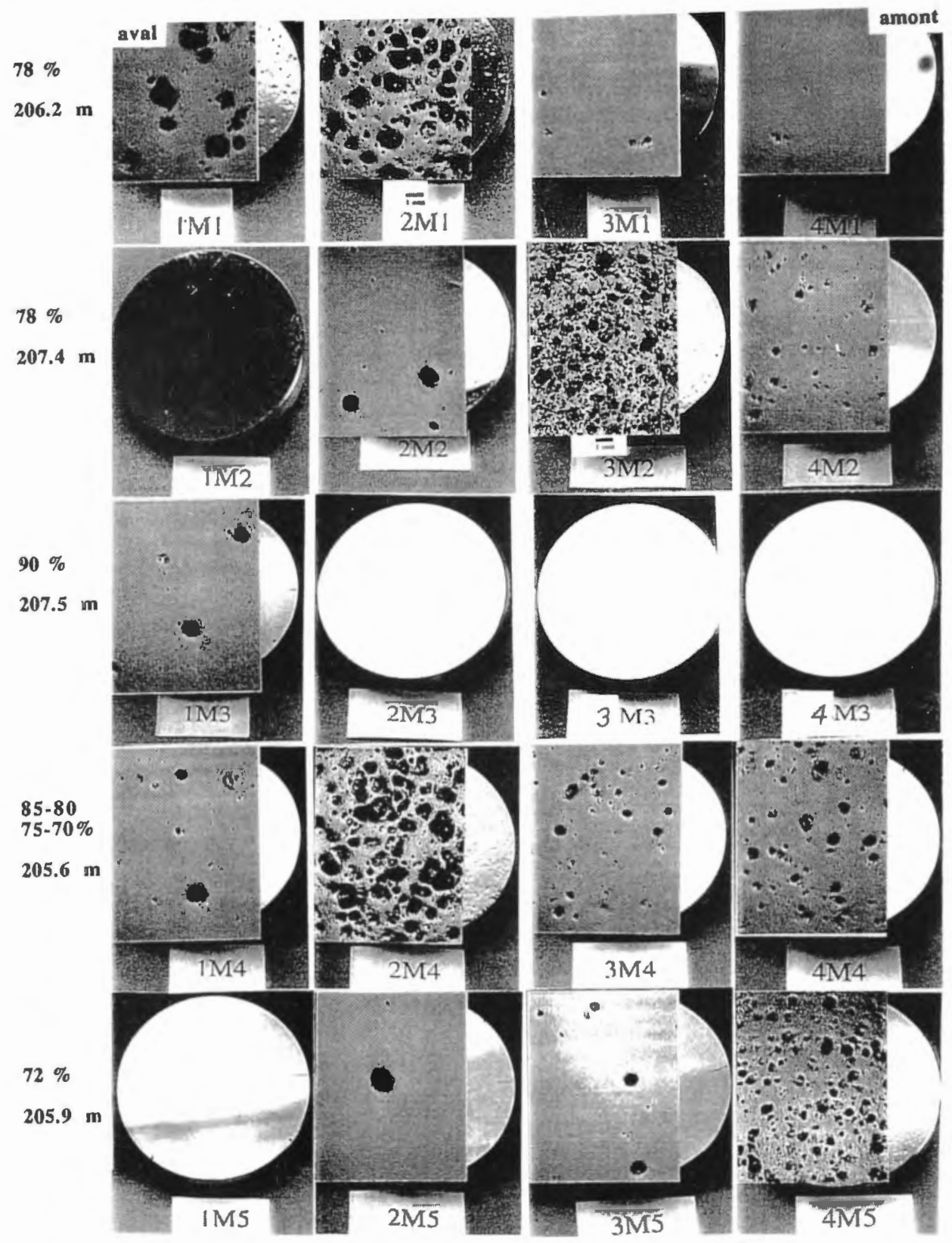

Figure 6. Pitted discs and 10X micrographs of pitted areas. 
These results are consistent with those of the previous campaign and show the combined effect of the increasing velocity with larger guide vane opening which promotes a more voluminous cavitation development and the adverse effect of a higher downstream level which contracts the cavitation development and forces implosions on the blade in the closure area of the cavitation cloud in the pressure recovery region.

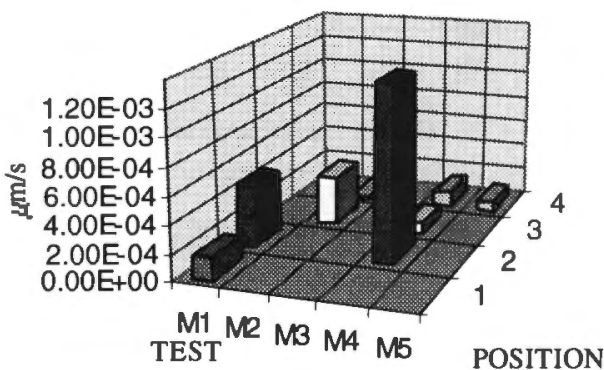

Figure 7. Volume pitting rate vs test and disc location

Since larger developments generate bigger shedded vortices, larger pits are found closer to the trailing edge of the blade. These observations are summarized in Figures 7 and 8 which show respectively the Volume pitting rate $\mathrm{V}_{\mathrm{d}}$ (total volume of pits per unit area and time) and the characteristic radius $\mathbf{R}_{v} \quad$ (average of pit radius weighted by $V_{i}$, the pit volume). The data for these figures are obtained by scanning the pitted samples with an UBM Laser profilometer and processing the output files with the "Adresse" software developed for EDF by CREMHyG (2).

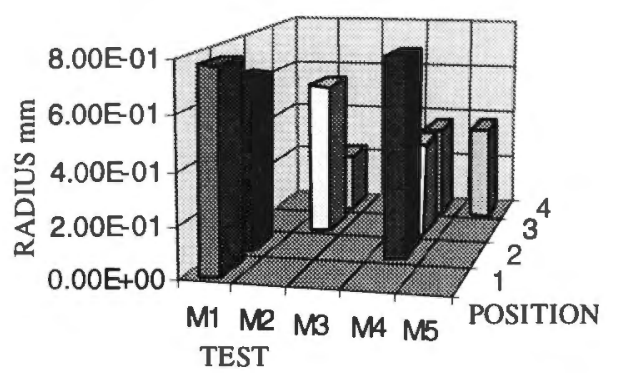

Figure 8. Characteristic radius Rv of the pits vs test and disc position on blade 4 .

These Figures clearly show that the largest Volume pitting rates are recorded in tests M1 and M4, the sweep test, in position 2 closer to the trailing edge. This is due to conditions at 80 and $75 \%$ guide vane opening which probably both contribute to pitting at this location. Individual pit diameters approaching 2 $\mathrm{mm}$, the largest we have ever observed, were found in test Ml. These pits are found downstream where the larger and more energetic vortices implode. This is consistent with the notion of erosive power (3) where the potential energy of each cavity scales with its volume. In test M3 at $90 \%$ guide vane opening, no pits were recorded on samples 2 to 4 and very few on sample 1 . Under these conditions implosions occur on the blade towards the trailing edge or in the flow beyond.

\subsubsection{DECER electrochemical erosion detection}

The DECER erosion detector located between samples 2 and 3 produced a localized maximum erosion rate at $75 \%$ guide vane opening in sweep test M4 with a low downstream level and a next higher value at $78 \%$ with a high downstream level in test M2. This is illustrated in Figure 9 which shows the DECER erosion current in $\mu$ a in relation to the guide vane opening and downstream level. This current is proportional to the actual erosion rate on the surface of the titanium sensor. 
The initial pressure tests, the pit counting tests and the DECER measurements all indicate a variable cavitation development on blade 4 affected both by guide vane opening and downstream level with maximum aggressiveness in the 75 to $80 \%$ span of guide vane opening.

\subsubsection{Vibration measurements}

Vibration and pressure measurements were made during all

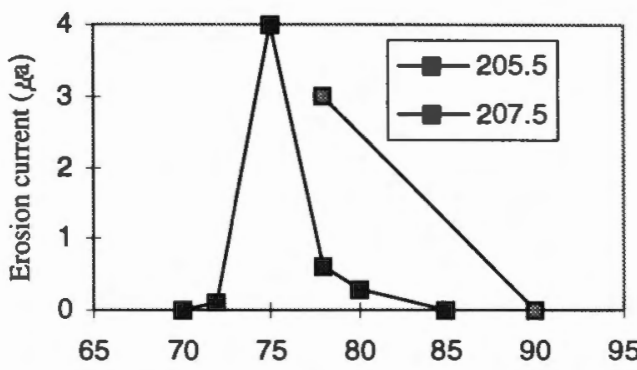

Guide vane opening (\%)

Figure 9. DECER erosion current vs guide vane opening tests. The blade mounted accelerometer was retained for these latter tests. The results are summarized in Figure 10. The three curves represent data normalized to the maximum observed value of each parameter during the low downstream level pressure and pit counting tests. The free symbols represent similar data for the high downstream level tests of both types.

Impacts were found to occur on the blade at or near a $60 \mathrm{~Hz}$ rate which corresponds to the guide vane passing frequency $(20$ guide vanes and $3 \mathrm{~Hz}$ rotation frequency) as indicated by the blade mounted accelerometer high frequency amplitude modulation envelope. The envelope of high frequency acceleration band pass filtered in a third octave bandwidth centered on 4 $\mathrm{kHz}$ was analyzed. Similar

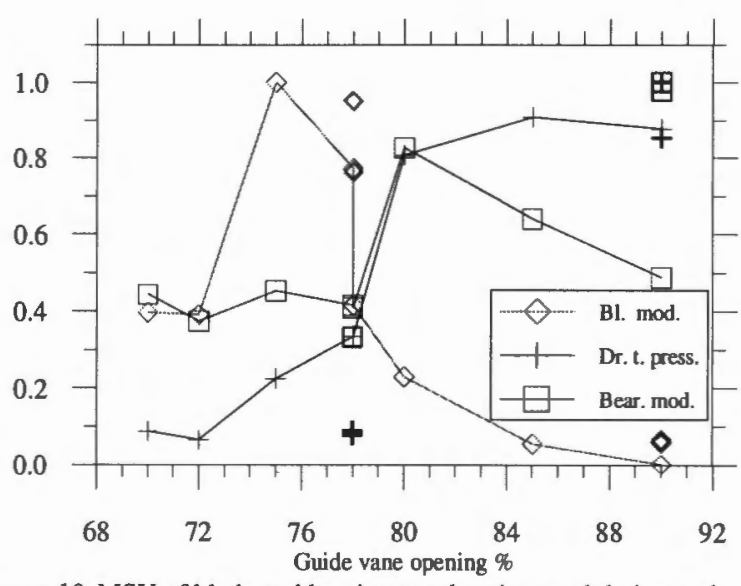

Figure 10. MSV of blade and bearing acceleration modulation and draft tube pressure around the guide vane passing frequency.

indications were obtained with the 0 degree upstream accelerometer at the lower guide bearing. In this case the 15 to $30 \mathrm{kHz}$ bandwidth was retained for analysis. These results agree with those of the 1993 tests. The three curves present respectively with diamonds, squares and crosses the Mean Square Value of modulation of the high frequency acceleration on the blade and at the lower guide bearing and the MSV of pressure pulsation above the draft tube access door. These parameters were calculated at $20+/-1$ and 2 times the runner rotation frequency over a quarter of a $\mathrm{Hz}$ bandwidth at each of these 5 frequencies.

These curves clearly show that maximum aggressiveness on blade 4 occurs around 75 to $80 \%$ G.V. opening depending on downstream level. A higher downstream level displaces the high intensity points towards the greater G.V. openings. The indications at the lower guide bearing are different with maximum values occurring at $80 \%$ G.V. 
opening for low downstream levels and $90 \%$ for high downstream levels. The guide bearing sensor modulation envelope is in good agreement with the indications of the draft tube pressure sensor. As opposed to the blade mounted accelerometer which is affected essentially by local events, the response of this pressure sensor, like the guide bearing accelerometer, is determined by the overall runner cavitation performance.

\section{Discussion and conclusions}

The availability of tools to characterize the aggressiveness of cavitation at the prototype level has been demonstrated in this test program. Localized pressure, pitting, DECER electrochemical erosion detection and vibration measurements have all concurred to characterize the cavitation behavior of blade 4 of this Francis prototype. Increasing guide vane openings or lower downstream levels produce a larger cavitation development and greater aggressiveness inasmuch as the implosions do not occur beyond the trailing edge of the blade. Overall vibration measurements at the guide bearing and pressure measurements at the draft tube reveal maximum aggressiveness at the maximum tested guide vane opening and downstream level. This suggests that all blades do not behave identically under the same hydraulic conditions from a cavitation point of view and that maximum overall aggressiveness occurs under high flow velocity and downstream levels which produce the best conditions for high erosive power of the cavitating flow. A set of blades with high erosion, of which blade 4 is typical, go into cavitation early and are followed later by the others when hydraulic conditions are more severe. This explains the heterogeneous blade erosions that have historically been observed on this machine. Because of this behavior, the transposition of these results to the model level may not be simple. However, the tools required to characterize quantitatively cavitation aggressiveness at the prototype level have been demonstrated and data is available for comparisons with those obtained in the model test program (4). In closing, the authors would like to thank Louis Bezençon and Georges Jotterand of IMHEF and Pierre Lavigne and Jacques Larouche of IREQ for their fundamental contributions to the success of this program.

\section{References}

1. Bourdon, P., Simoneau R., and Dorey, J-M., "Accelerometer and Pit Counting Detection of Cavitation Erosion on a Laboratory Jet and a Large Francis Turbine", Proceedings of the XVII IAHR Symposium, Beijing, China, 1994, Vol. 2, pp. 599-615.

2. Fortes -Patella $\mathbf{R}$ and Reboud, J.L., "Analysis of cavitation erosion by numerical simulation of solid damage", Proceedings of the XVI IAHR Symposium, Sao Paulo, Brazil, 1992, Vol. 2, pp.617-626.

3. Farhat, M., Pereira, F. and Avellan F., "Cavitation Erosion Power as a Scaling Factor for Cavitation Erosion in Hydraulic Machines", Proceedings of the ASME Symposium on Bubble Noise and Cavitation Erosion in Fluid Systems, ASME Fed-Vol. 176, pp. 95-104, New-Orleans, USA, Dec. 1993.

4. Caron, J.-F., et al., "Cavitation Erosion Prediction on Francis Turbines, Part II: Model Tests and Flow Analysis", paper to be presented at the XVIII IAHR Symposium, Section on Hydraulic Machinery and Cavitation, Valencia, Espania, September 1996. 